\title{
Ângulo de deflexão e fluxo de calor convectivo em sistema defletor do jato de exaustão de combustível sólido em plataforma de lançamento
}

\section{Deflection angle and convective heat flow in jet exhaust deflector system of solid fuel in launch pad.}

Élcio Nogueira ${ }^{1}$

Paulo Gilberto de Paula Toro ${ }^{2}$

Artigo

Original

Original

Paper

\begin{abstract}
Palavras-chave
Combustível sólido

Efetua-se uma análise dos principais parâmetros responsáveis pela troca de calor quando um jato de combustão supersônico, constituído de gás e partículas de alumina, incide sobre a superfície inclinada

Plataforma de lançamento do defletor de jato em uma plataforma de lançamento. Os resultados obtidos demonstram que, com relação ao ponto de deslocamento

Fluxo Convectivo da onda de choque, é possível utilizar ângulos de incidência mais acentuados quando os gases de exaustão contem uma fração de meio

Onda de choque sólido. Este é um resultado que tem consequências práticas de extrema importância: maiores ângulos de incidência do jato são desejáveis, uma

Escoamento supersônico vez que contribuem para uma base de lançamento menos elevada, e se traduzem em menor custo no projeto da plataforma.
\end{abstract}

\section{Abstract}

An analysis of the main parameters responsible for the heat exchange when a jet of supersonic combustion, consisting of gas and alumina particles, focuses on the inclined surface of the deflector jet on a launch pad is carried out. The results show that, concerning the place of displacement of the shock wave, it is possible to use the most marked angles of incidence when the exhausting gases contains a fraction of a medium solid. This is a result that presents very important practical consequences: increased angles of incidence of jet are desirables, once they contribute to a lower launch platform and therefore less cost to the platform project.
Key words

Solid combustion

Launch platform

Convective flow

Shock wave

Supersonic flow
Recebido em $04 / 2012$

Aprovado em 08/2012

\footnotetext{
${ }^{1}$ Centro Universitário de Volta Redonda - UniFOA - Dep. de Engenharia de Produção

${ }^{2}$ Instituto de Estudos Avançados - IAE/CTA
} 


\section{Introdução}

No projeto de veículos espaciais a análise térmica consiste em se identificar as principais fontes de aquecimento externo e, posteriormente, desenvolver modelos teóricos e experimentais que permitam avaliar quantitativamente os níveis de aquecimento sobre regiões críticas do veículo. Uma destas fontes de aquecimento consiste dos processos convectivos e radiativos, devido aos gases de exaustão a elevadas temperaturas na plataforma de lançamento. De fato, já foi demonstrado [Carvalho et al., 1990] que a temperatura na superfície do veículo, no instante do lançamento, depende do nível de temperatura dos gases de exaustão que se encontram próximo a ele. Um fluxo não controlado de gases de exaustão, provenientes do motor de um foguete, no momento do lançamento de um veículo, pode causar sérios riscos para o mesmo e para a plataforma de lançamento.

Torna-se necessário implantar métodos para controlar e dissipar a grande quantidade de energia emitida pelos gases de exaustão, com o objetivo de promover, principalmente a integridade do veículo. Um dos métodos de proteção é o que utiliza um sistema defletor de jato (Figura 01), que é um dispositivo que permite desviar o jato dos gases de exaustão para fora da área de lançamento.

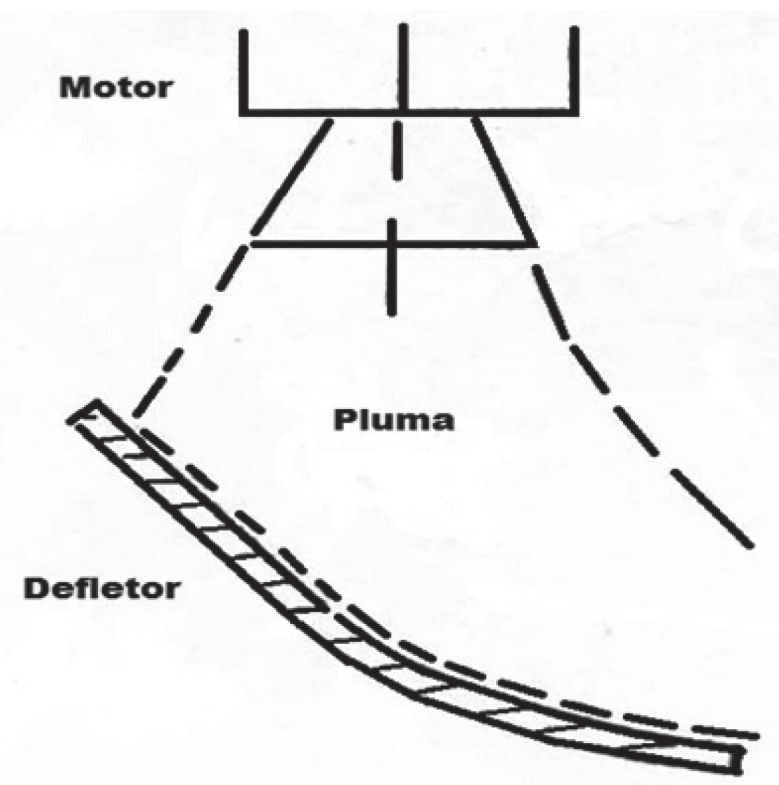

Figura 01 - Defletor de Jato

Quando os gases de exaustão incidem sobre a superfície defletora, a velocidade do jato decresce, ao mesmo tempo em que ocorre um aumento da temperatura e pressão. O ângulo de incidência do jato sobre a superfície determina o decréscimo na velocidade. Poucos materiais têm propriedades físicas e mecânicas para resistir a elevadas temperaturas e pressões. Prevenir a integridade do defletor é um sério desafio.
Neste trabalho, com o objetivo de possibilitar a determinação aproximada do fluxo de calor convectivo sobre a superfície do defletor, efetua-se uma análise dos principais parâmetros responsáveis pela troca de calor quando um jato supersônico, constituído de gás e partículas de alumina, incide sobre uma placa plana inclinada (Figura 02). 


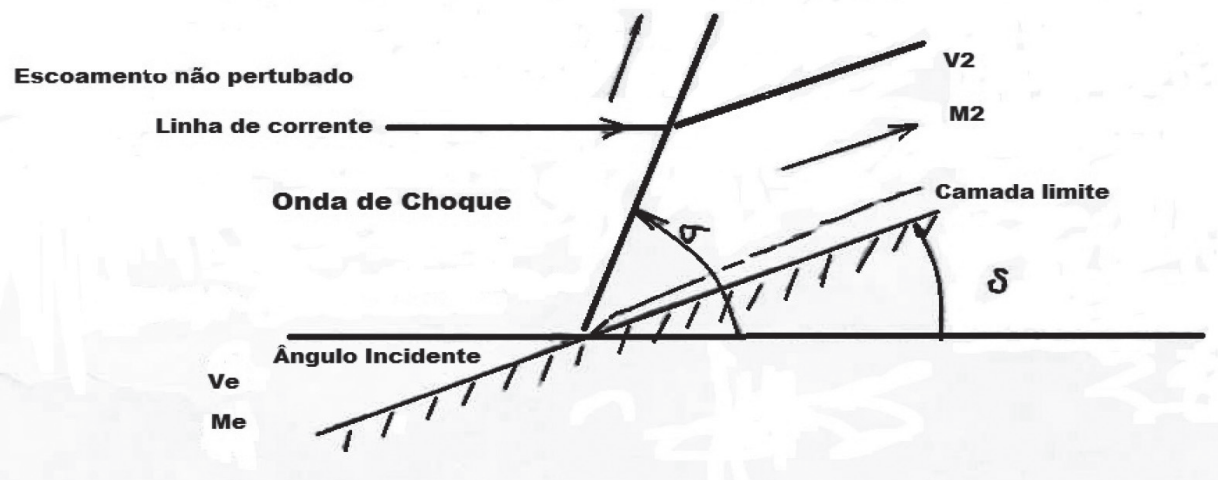

Figura 02 - Jato Supersônico incidindo sobre Placa Plana Inclinada

\section{Análise}

É evidente que o grande problema associado ao defletor encontra-se na alta transferência de energia, na forma de calor, dos gases de exaustão para a superfície defletora, e a capacidade limitada desta superfície em remover ou absorver, rapidamente, esta energia, de forma a prevenir a sua integridade física. A fonte do problema em questão encontra-se na determinação do coeficiente de transferência de calor na parede do defletor que, a altas velocidades, é influenciado pela onda de choque, pelo ângulo de incidência do jato e pelas perturbações ao longo da camada limite, que se formam quando o jato incide sobre a superfície defletora. No ponto de impacto do jato sobre a superfície (denominado ponto de estagnação) a espessura da camada limite é desprezível, e o coeficiente de transferência de calor é máximo. Neste caso [Jeeps e Robson, 1967], o coeficiente de transferência de calor pode ser determinado para pontos muito próximos do ponto de estagnação, para um jato sem partículas sólidas, através da expressão:

$$
\frac{h(x) x}{K}=0.042 R_{e}(x)^{4 / 5} P_{r}^{1 / 3}
$$

onde

$$
R_{e}(x)=\frac{\rho_{g} V_{x} x}{\mu}
$$

e

$$
P_{r}=\frac{C_{p g} \mu}{K}
$$

em que:

$\mathrm{x}$ - distância ao longo da placa, em relação ao ponto de impacto

$\mathrm{k}$ - condutividade térmica do gás

$\rho_{\mathrm{g}}$ - densidade do gás

$\mathrm{V}_{\mathrm{x}}$ - velocidade do gás, paralela à superfície da placa

$\mu$ - viscosidade do gás

$\mathrm{C}_{\mathrm{pg}}$ - calor específico do gás

O maior dano, em termos do processo de transferência de calor, deve ocorrer onde o coeficiente de transferência de calor é máximo. Entretanto, mesmo para pontos relativamente distantes do ponto de estagnação, o coeficiente de transferência de calor, devido às altas velocidades envolvidas, é muito elevado e, neste caso, pode ser estimado pela expressão apresentada por Evans e Sparks (1963):

$$
\frac{h(x) x}{K}=0.0296 R_{e}(x)^{4 / 5} P_{r}^{1 / 3}
$$

Equação 04

O coeficiente de transferência de calor é fortemente dependente das propriedades do jato incidente, e estas, por sua vez, dependem da velocidade do jato no ponto de impacto e do ângulo de incidência, em relação 
à superfície defletora, como mostra a Figura 02. A quantidade de calor transferido para a superfície do defletor é função do ângulo de incidência e do tempo de exposição aos gases de exaustão. À medida que o tempo de exposição aumenta menores ângulos de incidência são requeridos para reduzir a transferência de calor. Entretanto, desde que menores ângulos de incidência aumentam a altura do defletor e, logicamente, a altura da base de lançamento, é desejável empregar o maior ângulo de incidência possível.

Uma onda de choque oblíqua, do tipo representado através da Figura 02, é produzida quando um jato supersônico incide sobre uma superfície inclinada. Por considerações geométricas e aerodinâmicas, demonstra-se que a seguinte expressão possibilita determinar o ângulo de deflexão $(\sigma)$ que a onda de choque faz com a direção de incidência do jato:

$$
\frac{1}{M_{e}{ }^{2}}=\sin ^{2} \sigma-\frac{\gamma+1}{2} \frac{\sin \sigma \sin \delta}{\cos (\delta-\sigma)}
$$$$
\text { Equação } 05
$$

$\mathrm{M}_{\mathrm{e}}$ é o número de Mach associado ao jato incidente no momento do impacto. Neste caso, desde que se conheça o ângulo de incidência $(\delta)$ do jato e a velocidade de impacto $\left(V_{e}\right)$, pode-se determinar a pressão, a temperatura $\mathrm{e}$ o número de Mach, após a onda de choque, através das seguintes expressões:

$$
\frac{P_{2}}{P_{e}}=\frac{2 \gamma}{\gamma+1}\left(M_{e} \sin \sigma\right)^{2}-\frac{\gamma-1}{\gamma+1}
$$

Equação 06

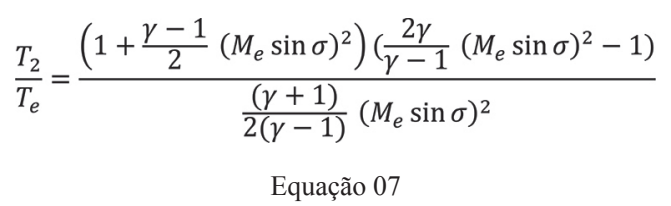

$$
\begin{gathered}
M_{2}^{2}=\frac{\left(M_{e} \sin \sigma\right)^{2}+\frac{2}{\gamma-1}}{\frac{2 \gamma}{\gamma-1}\left(M_{e} \sin \sigma\right)^{2}-1}\left(\sin ^{2}(\sigma-\delta)\right)^{-1} \\
\text { Equação } 08
\end{gathered}
$$

Onde $\gamma$ é a razão entre os calores específicos à pressão e a volume constante. O fluxo transferido do gás para a superfície é dado por:

$$
Q(x)=H(x)\left(T_{\text {estag }}-T_{w}(x, t)\right)
$$

Equação 09

para pontos próximos à região de estagnação, ou

$$
Q(x)=H(x)\left(T_{r}-T_{w}(x, t)\right)
$$

para pontos fora da região de estagnação, onde

$$
\begin{gathered}
T_{\text {estag }}=T_{e}+\frac{V_{e}^{2}}{2 C_{p g}} \\
\text { Equação 11 } \\
T_{r}=T_{2}\left(1+R\left(\frac{\gamma-1}{2}\right) M_{2}^{2}\right)
\end{gathered}
$$$$
\text { Equação } 12
$$

em que R é o fator de recuperação.

O efeito da variação das propriedades pode ser incluído, de maneira aproximada (Eckert, 1956), nas relações de transferência de calor acima, se estas forem avaliadas à temperatura de recuperação $T_{r}$.

\section{Método de Solução}

O procedimento adotado na determinação do coeficiente de transferência de calor, por intermédio da Equação 01, quando os produtos de combustão são constituídos de gases, é similar aos procedimentos preconizados por Evans e Sparks (1963) e Jeeps e Robinson (1966). Entretanto, para melhor compreensão da aproximação efetuada para escoamento bifásico, sintetizamos os passos adotados:

Passo 1 - Determinação do ângulo de deflexão (ó) Passo 2 - Determinação de $P_{2}, T_{2}$ e $M_{2}$ Passo 3 - Determinação das temperaturas de estagnação $\left(T_{\text {estag }}\right)$ e recuperação $\left(T_{r}\right)$

Passo 4 - Determinação do fluxo de calor, com propriedades determinadas através de $T_{r}$ e $P_{2}$. 
Neste caso assumimos um procedimento aproximado, supondo que a mistura se comporta como um pseudo-gás (Wallis, 1969), e as propriedades são determinadas através das relações:

$$
\bar{R}_{e}=\frac{\bar{R}}{1+\lambda}
$$

Equação 13

$$
C_{e}=\frac{C_{p g}+\lambda C_{s}}{1+\lambda}
$$

Equação 14

$$
\gamma_{e}=\frac{1+\lambda \frac{C_{s}}{C_{p g}}}{\frac{1}{\gamma}+\lambda \frac{C_{s}}{C_{p g}}}
$$

Equação 15

Em que $\overline{\mathrm{R}}$ é a constante do gás, é o calor específico do sólido e a fração em massa das partículas. Salientamos que o meio sólido é fortemente dependente da temperatura e, neste caso, utilizamos a expressão fornecida por Pessoa Filho e Cotta (1989):

$$
\begin{gathered}
C_{S}=41.063\left(22.08+0.008971 T-522500 T^{2}\right) \\
\text { Equação } 16
\end{gathered}
$$

A viscosidade do pseudo-gás é determinada através da equação de Einstein (Wallis, 1969):

$$
\mu_{e}=\mu_{g}(1+2.5 \alpha)
$$

onde

$$
\alpha=\frac{\lambda \rho_{e}}{(1+\lambda) \rho_{S}}
$$

Equação 18

\section{Resultados e Discussões}

Para obtenção de resultados através da análise efetuada acima, utilizamos o procedimento adotado por Machado et al. (1991), admitindo-se um prolongamento da tubeira do primeiro estágio do Veículo Lançador de Satélite - VLS, e obtivemos valores aproximados para as velocidades, temperaturas e pressões a uma distância adimensional (X/Re) do divergente, em que $\mathrm{X}$ é a distância do ponto de impacto e Re o raio do divergente.

Através da Figura 03 analisamos os efeitos do ângulo de incidência e da fração em massa no coeficiente de transferência de calor médio [hmédio $(\mathrm{x})$ ], a uma distância próxima do ponto de impacto, em que

$$
h_{\text {médio }}=\frac{1}{x} \int_{0}^{x} h(x) d x
$$

$$
\text { Equação } 19
$$

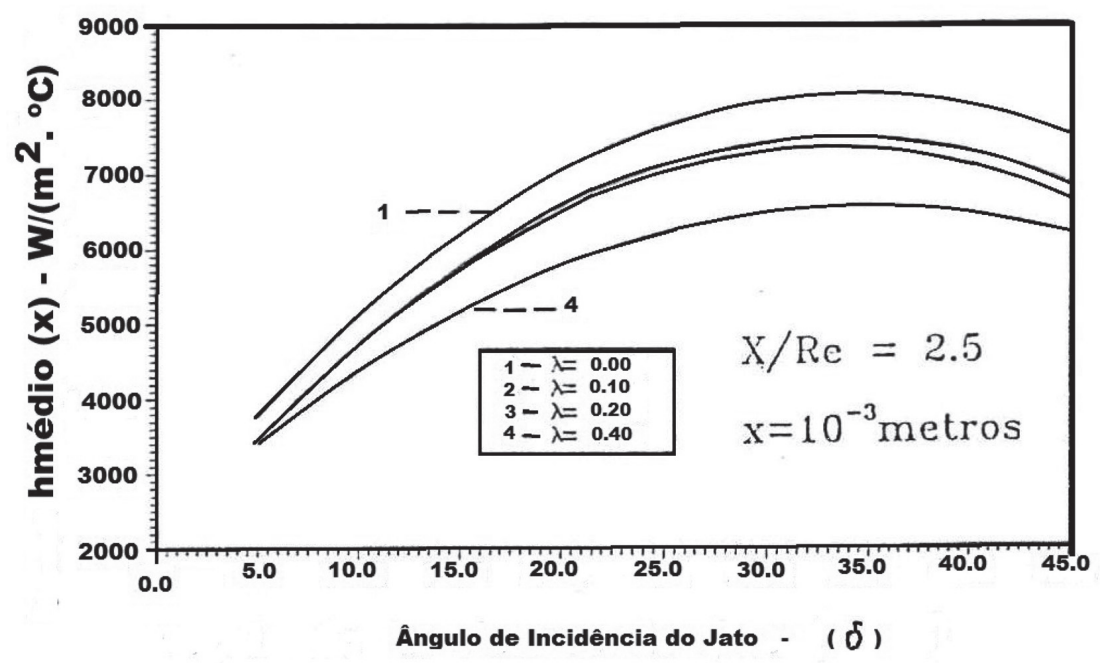

Figura 03 - Coeficiente de transferência de calor médio, a uma distância de 1 mm em relação ao ponto de impacto do jato, situado a uma distância igual a 2.5 vezes o raio do divergente. 
O coeficiente de transferência de calor médio [hmédio $(\mathrm{x})$ ] cresce à medida que o ângulo de incidência aumenta, até um valor próximo de $35^{\circ}$, para todas as frações em massa analisadas. A partir deste limite, para todas as situações, o coeficiente de transferência de calor sofre um decréscimo. Observa-se que o coeficiente de transferência decresce, para um dado ângulo de incidência, quando se aumenta a fração em massa do meio sólido.

A Figura 04 mostra que o coeficiente de transferência de calor é extremamente elevado em regiões próximas ao ponto de impacto e, à medida que se distancia deste ponto, decresce rapidamente para valores relativamente baixos. Isto demonstra que, de acordo com Evans e Sparks (1963) e Jeeps e Robinson (1967), preservar a integridade do material do defletor é, de fato, um sério desafio. Observa-se que o coeficiente de transferência de calor é significativamente inferior, na região próxima ao ponto de impacto, quando se considera meio sólido de $40 \%(\lambda=0.40)$ de fração de massa, em relação ao escoamento de gás $(\lambda=0.00)$.

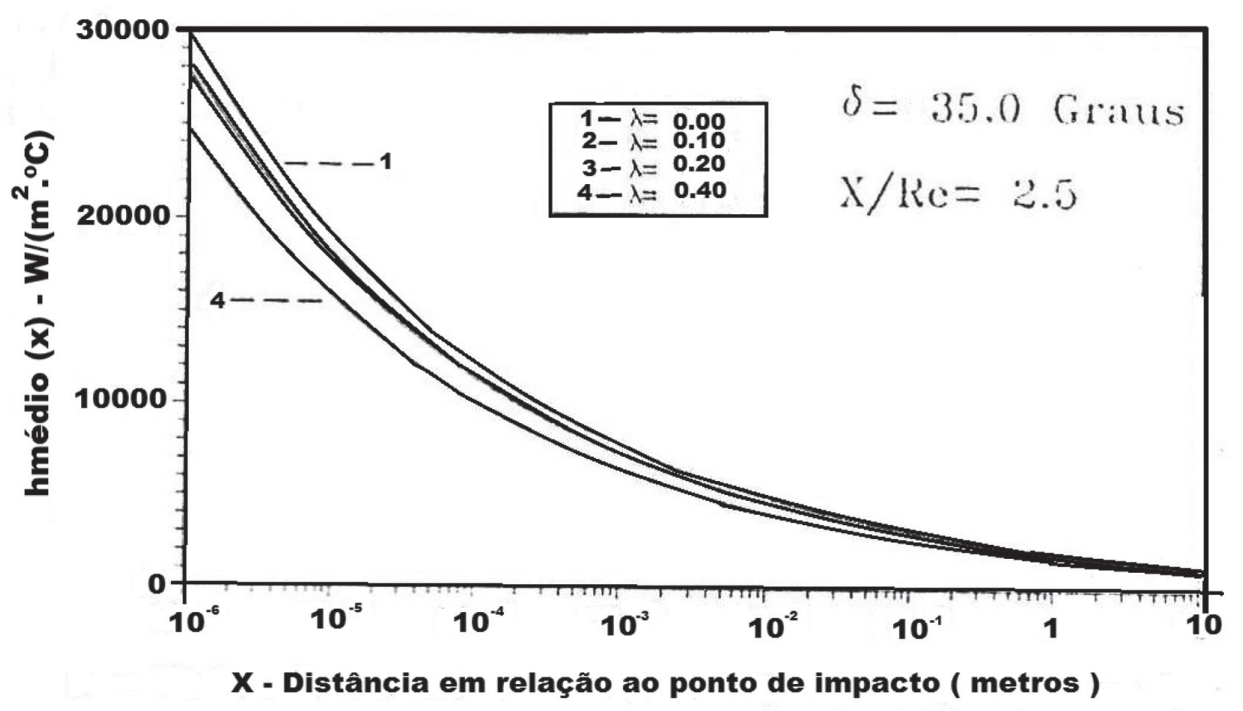

Figura 04 - Coeficiente de transferência de calor médio, função da distância do ponto de impacto, para ângulo de incidência onde ocorre a máxima taxa de transferência de calor.

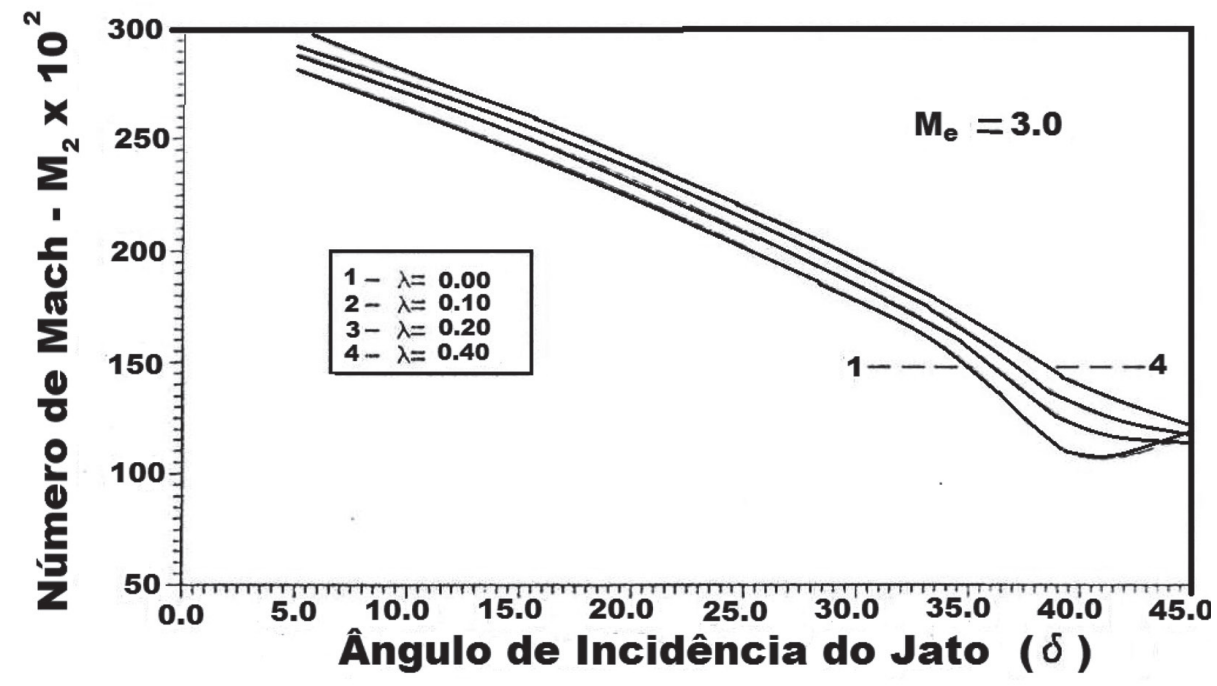

Figura 05 - Número de Mach após a Onda de Choque $\left(\mathrm{M}_{2}\right)$ 
Os resultados verificados através das Figuras 03 e 04 podem ser justificados quando analisamos as Figuras 05 e 06. Nestes casos, salientamos que os resultados obtidos para escoamento de gás $(\lambda=0.00)$ são absolutamente compatíveis aos resultados obtidos por Evans e Sparks (1963), e demonstram que ocorre um deslocamento da onda de choque para um ângulo de incidência próximo de $45^{\circ}$. Assim, observa-se, Figura 06, uma progressiva descompres- são do gás para ângulos de incidência a partir de $35^{\circ}$, o que justifica o decréscimo observado para o coeficiente de transferência de calor.

Para frações em massa do meio sólido diferente de zero observa-se, devido à maior inércia das partículas e da relativa incompressibilidade, que o efeito do deslocamento é retardado, contribuindo para uma variação menos acentuada no coeficiente de transferência de calor entre $35^{\circ}$ e $45^{\circ}$ do ângulo de incidência.

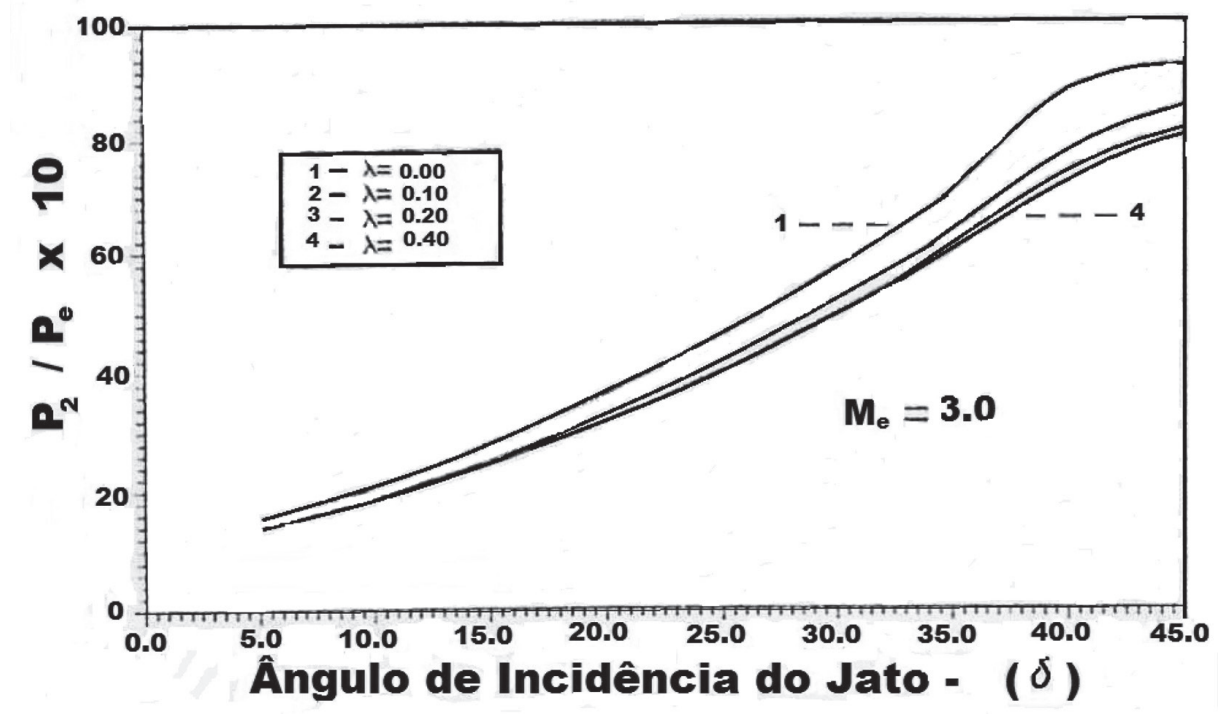

Figura 06 - Razão de pressão, entre choque e escoamento incidente, para número de Mach 3.0

Os resultados apresentados através da Figura 07 demonstram que, com relação ao ponto de deslocamento da onda de choque, é possível utilizar ângulos de incidência mais acentuados quando os gases de exaustão contêm uma fração de meio sólido.

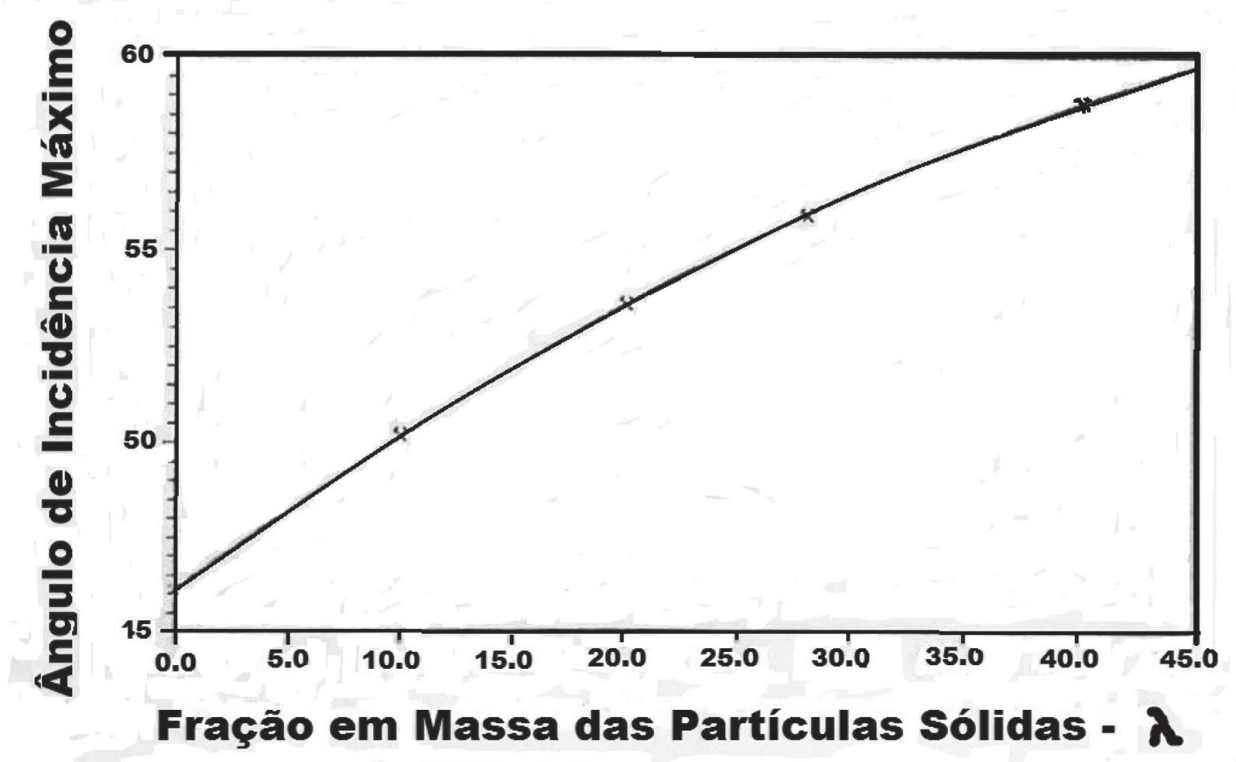

Figura 07 - Ângulo de Incidência máximo, acima do qual a onda de choque separa da superfície, permitindo o retorno dos gases de exaustão. 
As propriedades dos meios, assim como dados relevantes considerados para obtenção dos resultados, foram:

$R=0.9$

$\bar{R}=332.57 \frac{J}{\left(K_{g} K\right)}$

$\gamma=1.2079$

$\rho_{S}=3990 K_{g} / m^{3}$

$r_{P}($ raio da partícula $)=3.010^{-6} \mathrm{~m}$

$W($ vazão em massa total $)=120 \mathrm{Kg} / \mathrm{s}$

Apesar do raio da partícula não aparecer explicitamente na análise, o coeficiente de transferência de calor obtido é dependente deste parâmetro, uma vez que a velocidade, a temperatura e a pressão no ponto de impacto dependem dele.

\section{Conclusões}

O coeficiente de transferência de calor convectivo na superfície do Defletor de Jato, quando submetido a um jato bifásico dos gases de exaustão, é inferior ao fluxo de calor obtido para um jato constituído apenas de gases.

$\mathrm{O}$ ângulo de incidência máximo, para o qual ocorre um refluxo dos gases de exaustão, é maior para o jato bifásico. Os resultados obtidos demonstram de forma clara que, para gases de exaustão oriundos de combustível sólido, a plataforma de lançamento pode ter altura inferior ao da plataforma utilizada para gases de combustão ausentes de partículas sólidas, refletindo-se no custo final do projeto da mesma.

Não há trabalho de análise similar em literatura aberta. Uma vez que a literatura aberta para este tipo de problema é inexistente, sugerem-se, para a completa validação dos resultados obtidos através da presente análise, que se realizem ensaios experimentais que permitam estimar o coeficiente de transferência de calor.

\section{Agradecimento}

Nogueira, E. agradece ao Instituto de Aeronáutica e Espaço - IAE, na pessoa do Dr. Paulo Moraes Junior, a oportunidade de efetuar este trabalho.

\section{Referências}

1. EUGENE L. FLEEMAN. Technologies for Future Precision Strike Missile Systems - Missile/Aircraft Integration. Paper presented at the RTO SCI Lecture Series on "Technologies for Future Precision Strike Missile Systems", held in Tbilisi, Georgia, 18-19 June 2001.

2. ALEX WILEY, RAJAN KUMAR AND FARRUKH ALVI. Noise and Flow field Characteristics of a Supersonic Jet Impinging on a Porous Surface. 48th AIAA Aerospace Sciences Meeting Including the New Horizons Forum and Aerospace Exposition 4 - 7 January 2010, Orlando, Florida.

3. A. PAUL, D.D. JAYASEELAN, S. VENUGOPAL, E. ZAPATA-SOLVAS, J. BINNER, B. VAIDHYANATHAN, A. HEATON, P. BROWN AND W. E. LEE. UHTC composites for hypersonic applications - Hypersonic aircraft like DARPA's Falcon HTV-2 operate under extreme conditions and demand materials that can handle high temperatures and mechanical and shock wave loads. American Ceramic Society Bulletin, Vol. 91, No. 1. January - February 2012.

4. H.-Y. KIM, Z. C. FENG, AND J.H. CHUN. Instability of a liquid jet emerging from a droplet upon collision with a solid surface. Physics of Fluids. Volume 12, Number 3. March 2000.

5. RASHID A. AHMAD. Convective Heat Transfer in the Reusable Solid Rocket Motor of the Space Transportation System. Thiokol Propulsion, a Division of ATK Aerospace Company Inc., Published by the American Institute of Aeronautics and Astronautics. 2002.

6. ANDERSON JR., J. D. Modern Compressible Flow - With Historical Perspective. Second Edition. Aerospace Series. International Edition 1990. 
7. CARVAlho, T. M. B.; COTTA, R. M.; PESSOA FILHO, J. B. Análise Térmica da Região da Base de Veículos Espaciais durante a Partida e Vôo Propulsado a Grandes Altitudes. III Encontro Nacional de Ciências Térmicas ENCIT, Itapema-SC, 1990.

8. JEPPS, G.; ROBINSON. M. L. Convective Heating at the Deflecting Surface of a Rocket Launch-Pad. Journal of the Aeronautical Society, Vol. 71, pp. 469-475, 1967.

9. EVANS, R. L.; SPARKS, O. L. Launch Deflector Design and their Application. NASA TN D-1275, 1963.

10. ECKERT, E. R. G. Engineering Relations for Heat Transfer and Friction in HighVelocity Laminar and Turbulent Boundary Layer Flow over Surface with Constant Pressure and Temperature. Trans. ASME, Vol. 78, pp. 1273-1284, 1956.

11. WALliS, G. B. One Dimensional TwoPhase Flow. McGraw-Hill Company, 1969.

12. PESSOA FILHO, J. B.; COTTA, R. M. Radiação Térmica Proveniente da Pluma de Foguete a Propelente Sólido. Anais do III Workshop de Combustão e Propulsão, PP. 51-62, Lorena, 1989.
13. MACHADO, H. M.; COTTA, R. M.; PESSOA FILHO, J. B. Evaluation of Velocity and Temperature Lags in Two Phase Flow Trough the Nozzle of a Solid Propellant Rocket Motor. Anais do XI Congresso Brasileiro de Engenharia Mecânica, São Paulo - SP, 1991.

14. W. D. LANNING, F. T. HUNG. Shuttle ascent and shock impingement aerodynamic heating studies. National Aeronautics and Space Administration George C. Marshall Space Flight Center Aero-Astrodynamics Laboratory under contract NAS8-26268. FINAL REPORT December 1971.

15. JOHN N. B. LIVINGOOD, PETER HRYCAK. Impingement Heat Transfer from Turbulent Air Jets to Flat Plates A Literature Survey. Lewis Research Center National Aeronautics and Space Administration Cleveland, Ohio 44135. National Aeronautics and Space Administration Washington, D. C. 20546. May 1973.

16. ARANTES, F. S; NOGUEIRA, E. Determinação do Ângulo de Deflexão de uma Onda de Choque em Escoamento Hipersônico. Relatório Parcial de Bolsa de Iniciação Científica (CNPq - Processo: 523320/96-5 (NV)). Maio Dezembro de 1997.

Endereço para Correspondência:

Élcio Nogueira - Professor Assistente Dr. UniFOA elcionogueira@hotmail.com

Centro Universitário de Volta Redonda - UniFOA Campus Universitário Olezio Galotti

Av. Paulo Erley Abrantes, 1325

Três Poços - Volta Redonda - RJ

CEP: 27.240-560 